\title{
Optically stimulated luminescence signals under various stimulation modes assuming first-order kinetics
}

\author{
A. J. J. Bos ${ }^{1, *}$ and J. Wallinga ${ }^{2}$ \\ ${ }^{1}$ Faculty of Applied Sciences, Delft University of Technology, Mekelweg 15, NL 2629 JB Delft, The Netherlands \\ ${ }^{2}$ Netherlands Centre for Luminescence dating, Delft University of Technology, Mekelweg 15, NL 2629 JB Delft, The Netherlands
}

(Received 30 January 2009; revised manuscript received 1 April 2009; published 19 May 2009)

\begin{abstract}
We present mathematical descriptions of optically stimulated luminescence (OSL) signals under linearly, hyperbolically, exponentially, and reciprocally increasing stimulation intensity for a one-trap one-recombination-center model assuming charge transfer governed by first-order kinetics. Depending on the stimulation mode, the OSL signal can be monotonically increasing, monotonically decreasing, show a peak shape, or be constant. The shape of the OSL signal is controlled by a stimulation-rate parameter (governed by the stimulation mode) and a decay-rate parameter that is proportional to the photoionization cross section. We demonstrate that the luminescence signal as a function of time under exponentially increasing-optical stimulation (EM-OSL) shows the same evolution in time as the luminescence signal under hyperbolically increasingthermal stimulation (HM-TL). This similarity allows a new interpretation of the $I \cdot t$ versus In $t$ plot, where $I$ is the optically stimulated luminescence intensity and $t$ is the time. For a phosphor with several optically active traps, the OSL signal will contain several components. We show that the mathematical descriptions of the OSL signals under all stimulation modes can be related to the description of the OSL signal derived with continuous-wave (CW) stimulation. These so-called pseudo-OSL signals are helpful in a visualization of the various components in the OSL signal, where different pseudo-OSL transformations can be used to amplify different aspects. We demonstrate that the stimulation mode or pseudo-OSL transformation used has no effect on the overlap of the different OSL components. This implies that for the separation of multiple OSL components there is, in principle, no preference for a specific stimulation mode or pseudo-OSL transformation. Finally, we present a transformation related to hyperbolically modulated OSL which does facilitate separation of OSL components and may be of use for determining component specific photoionization cross sections or trapped-charge concentrations.
\end{abstract}

DOI: 10.1103/PhysRevB.79.195118

PACS number(s): 78.45.+h, 78.55.Hx, 78.60.Kn

\section{INTRODUCTION}

During exposure of a semiconductor or insulator to ionizing radiation, electron-hole pairs are created. Some of these are trapped in centers with energy levels in the band gap. Upon stimulation with photons of appropriate energy, the charge carriers can be delocalized and recombine at recombination centers. This results in the emission of light, a phenomenon called optically stimulated luminescence (OSL). Instead of photons, the charge carriers can also be freed from their traps by heating the sample. In this case, the phenomenon is called thermoluminescence (TL). The emitted light is a measure of the absorbed dose deposited during excitation of the sample, e.g., during the exposure to ionizing radiation. Highly sensitive TL and OSL materials have been developed for use in radiation dosimetry. ${ }^{1,2}$ OSL signals from natural materials, in particular, quartz and feldspar minerals, are widely used to determine the age of sediments ${ }^{3}$ and for accident dosimetry. ${ }^{4}$

OSL and TL techniques are also of great importance in material science, in particular, in the study of persistent luminescence ${ }^{5}$ and in the biomedical field ${ }^{6}$ where OSL is used for imaging. ${ }^{7}$ Another application of stimulated luminescence is in the development of optical memories. ${ }^{8}$ Storing data ("writing") into an OSL material is accomplished through trap filling with UV radiation and "readout" by measuring the infrared stimulated luminescence.

Usually, the OSL signal is measured using continuouswave $(\mathrm{CW})$ stimulation (CW-OSL), where the luminescence emission is recorded during stimulation with light of a certain wavelength and constant intensity. The monitored signal typically decays until all accessible traps are emptied and the luminescence ceases. In general, the shape of a CW-OSL signal is a rather featureless decaying curve. In the past, several attempts have been made to transform the signal into a peak-shaped curve which gives a more pronounced visualization of the components (corresponding to emission from different trapping centers) in the signal. Randall and Wilkins ${ }^{9}$ suggested plotting $I \cdot t$ as a function of $\ln t$, with $I$ as the intensity of the luminescent signal and $t$ is the time. Although they studied phosphorescence, the equations governing OSL and phosphorescence are very similar ${ }^{10}$ and therefore relevant to this study. Chen and Kristianpoller ${ }^{11}$ elaborated the Randall-Wilkins transformation and showed the similarity of the transformed signal to a thermoluminescence glow peak measured under hyperbolic heating. Kitis et al. ${ }^{12}$ applied the Randall-Wilkins transformation to CW-OSL decay curves and pointed out that in the transformed curve, it is easier to determine whether the trap is completely emptied. Moreover, the position of the maximum in the curve provides direct information on the decay constant.

In 1996 Bulur $^{13}$ introduced a new method of measuring OSL by ramping the stimulation intensity linearly in time, producing a linearly modulated (LM) optically stimulated luminescence (LM-OSL) signal. Later on, Bulur ${ }^{14}$ showed that this LM-OSL signal can also be obtained from the CWOSL signal by a simple mathematical transformation. Such a 
signal not obtained by the modulation of the stimulation intensity but through the transformation of the CW-OSL signal is called a pseudo-OSL signal. Another approach was described by Poolton et al., ${ }^{15}$ who obtained a pseudo-LM-OSL signal by ramping the sample period during $\mathrm{CW}$ stimulation. Chen and Pagonis ${ }^{10}$ derived yet another pseudo-LM-OSL signal for non-first-order kinetics in which the maximum is proportional to the initial concentration of charge carriers, which is-under appropriate conditions-proportional to the absorbed dose.

All these studies on stimulation modes and transformations aim to show the structure or a specific property of the OSL signal with greater clarity. Several studies claimed that one can resolve the contributions from the various traps more clearly with LM-OSL than with CW-OSL measurements. ${ }^{16,17}$ Mishra et al. ${ }^{18}$ posed that with nonlinear light modulation, there is also a better discrimination of the OSL components. Such discrimination of the OSL components is of relevance for studies of OSL materials as it would facilitate the quantification of trap parameters (trap depth photoionization cross section). For OSL dating using quartz, it is of paramount importance to isolate the fast OSL component from contributions of other components to the OSL signal. ${ }^{19}$ The reason for this is that the trap giving rise to the fast OSL component is the only trap in natural quartz that is rapidly reset upon light exposure and which is sufficiently stable to allow charge to remain trapped over geological time scales. The accuracy in the age determination would benefit greatly from improved extraction of the fast-component quartz OSL signal from the OSL contributions from other traps.

The relevance of component separation for OSL material studies and for luminescence dating inspired us to investigate a wide range of stimulation modes and mathematical transformations in a systematic way. In particular, we address the following questions: (i) is there an optical-stimulation mode that produces the same development in time of the luminescence intensity as the signal produced under thermal stimulation? (ii) Is there a specific stimulation mode that deserves preference for decomposing a multicomponent OSL signal? (iii) Are there specific advantages in the use of a pseudo-OSL signal?

First, we give the basic differential equation of the chargecarrier traffic and the assumptions on which this equation rests. From the differential equation follows the OSL signal under constant stimulation. Then we derive equations describing the OSL signals under linearly, hyperbolically (HM), exponentially (EM), and reciprocally modulated (RM) stimulation intensity. For each of the stimulation modes, we provide a pseudosignal, i.e., a transformation function that relates the modulated signal to the CW-OSL signal. We then use the derived equations to answer the research questions specified above and discuss implications for material studies and for luminescence dating.

\section{DESCRIPTION OF THE OSL-SIGNAL}

In this paper, a simplified model is used with only two energy levels in the band gap of the phosphor. One is acting as a trap where electrons are accumulated during exposure to ionizing radiation and the other as a hole trap that acts as a radiative recombination center. We assume that upon optical stimulation, only the electrons are freed from their traps and recombine with the holes with emission of light. If we assume (i) first-order kinetics (i.e., no retrapping) and (ii) detrapping probability increases linearly with stimulation intensity then the OSL signal $I_{\mathrm{OSL}}\left(\mathrm{m}^{-3} \mathrm{~s}^{-1}\right)$ can be described by ${ }^{20}$

$$
I_{\mathrm{OSL}}(t)=-\frac{d n}{d t}=n(t) \sigma(\lambda) \varphi(t),
$$

with $n(t)=$ trapped-electron concentration $\left(\mathrm{m}^{-3}\right)$ at time $t(\mathrm{~s})$, $\sigma(\lambda)=$ photoionization cross section $\left(\mathrm{m}^{2}\right)$ at stimulation wavelength $\lambda$, and $\varphi(t)=$ stimulation intensity at time $t$ in number of stimulation photons with wavelength $\lambda$ in $\mathrm{m}^{-2} \mathrm{~s}^{-1}$. We now describe the OSL signal under different stimulation modes.

\section{A. CW-OSL}

Under CW stimulation, the stimulation intensity $\varphi(t)$ is constant

$$
\varphi_{\mathrm{CW}}(t)=\varphi_{\mathrm{CW}} .
$$

We define the CW decay rate or detrapping parameter $\alpha\left(\mathrm{s}^{-1}\right)$ as

$$
\alpha=\sigma \varphi_{\mathrm{CW}} .
$$

Using this parameter in solving Eq. (1) with $\varphi(t)=\varphi_{\mathrm{CW}}$ gives

$$
I_{\mathrm{CW}}(t)=n_{0} \alpha \exp (-\alpha t),
$$

with $n_{0}$ as the initial trapped electron concentration at $t=0$. This is the well-known exponential decay with decay constant $\alpha$. For a system with several optically active traps (say $i$ ) and assuming no interaction between them, the OSL signal will be the sum of $i$ exponentials,

$$
I_{\mathrm{CW}}(t)=\sum_{i} n_{0, i} \alpha_{i} \exp \left(-\alpha_{i} t\right)
$$

For the area under each component $i$, independent of the decay rate, the following holds:

$$
\int_{0}^{\infty} I_{\mathrm{CW}, i}(t) d t=n_{0, i} .
$$

This provides a constraint for any transformation of $I_{\mathrm{CW}}$ : for any component, its time-integrated value should remain unaltered.

\section{B. OSL under modulated stimulation intensity}

In this section, we consider the case in which the stimulation intensity is not constant. Suppose that the stimulation intensity can be written as

$$
\varphi(t)=f(t) \varphi_{c},
$$

with $f(t)$ as a function that describes the time dependence of the intensity and $\varphi_{c}$ as a constant intensity. Solving the differential equation (1), with Eq. (7) results in 


$$
n(t)=n_{0} \exp \left\{-\sigma \varphi_{c} \int_{0}^{t} f(\theta) d \theta\right\},
$$

with $\theta$ as a dummy variable. Substituting Eq. (8) into Eq. (1) results in

$$
I_{\mathrm{OSL}}(t)=n_{0} \sigma \varphi_{c} f(t) \exp \left\{-\sigma \varphi_{c} \int_{0}^{t} f(\theta) d \theta\right\} .
$$

If $\varphi_{c}$ is taken equal to $\varphi_{\mathrm{CW}}$, this signal can be written in terms of the CW-OSL [Eq. (4)]. Such a signal is termed as a pseudo-OSL signal,

$$
I_{\text {pseudo-OSL }}(t)=f(t) I_{\mathrm{CW}}\left(t^{\prime}\right),
$$

with

$$
t^{\prime}=\int_{0}^{t} f(\theta) d \theta
$$

Equations (10) and (11) tell us that the intensity-modulated OSL signal can be found by first applying a time transformation $t \rightarrow t^{\prime}$. From the CW-OSL signal, the signal for $t^{\prime}$ is determined and the result multiplied by $f(t)$. Equation (10) shows that for any stimulation intensity function that can be described by Eq. (7), the intensity-modulated OSL signal can be derived from the CW-OSL signal. This will be further exemplified in the following sections.

\section{1. $L M-O S L$}

We consider a linearly increasing stimulation intensity according to

$$
\varphi_{\mathrm{LM}}(t)=\frac{\varphi_{P}}{P} t,
$$

with $\varphi_{P}\left(\mathrm{~m}^{-2} \mathrm{~s}^{-1}\right)$ as the intensity at the end of the stimulation period $P(\mathrm{~s})$. For this stimulation mode, we define the LM decay-rate parameter $\beta\left(\mathrm{s}^{-1}\right)$ as

$$
\beta=\sigma \varphi_{P} .
$$

The LM-OSL signal follows from Eq. (9) with $f(t)=t / P$, $\varphi_{c}=\varphi_{P}$, and using Eq. (13):

$$
I_{\mathrm{LM}}(t)=n_{0} \frac{\beta}{P} t \exp \left\{-\frac{1}{2} \frac{\beta}{P} t^{2}\right\} .
$$

This signal has a peak shape with a maximum at $t_{\max }$ $=\sqrt{P / \beta}$ and $I_{\max }=n_{0} \sqrt{\beta /(e P)}$ (see Fig. 1). The peak has an asymmetric peak shape with a steeply rising left-hand side and a relatively slowly decreasing right-hand side. We define an asymmetry factor $\tau=\kappa / \mathrm{FWHM}$ with $\kappa=t_{2}-t_{\max }$ or the high time half width, FWHM=the full width at half maximum $=t_{2}-t_{1}$ with $t_{\max }, t_{1}$, and $t_{2}$ as the maximum value and the two half-intensity values of the peak, respectively. It can be derived that FWHM $=1.631 / \sqrt{\beta / P}$ and the asymmetry factor $\tau=0.565$.

Pseudo-LM-OSL. The pseudo-LM-OSL signal follows from Eq. (10):

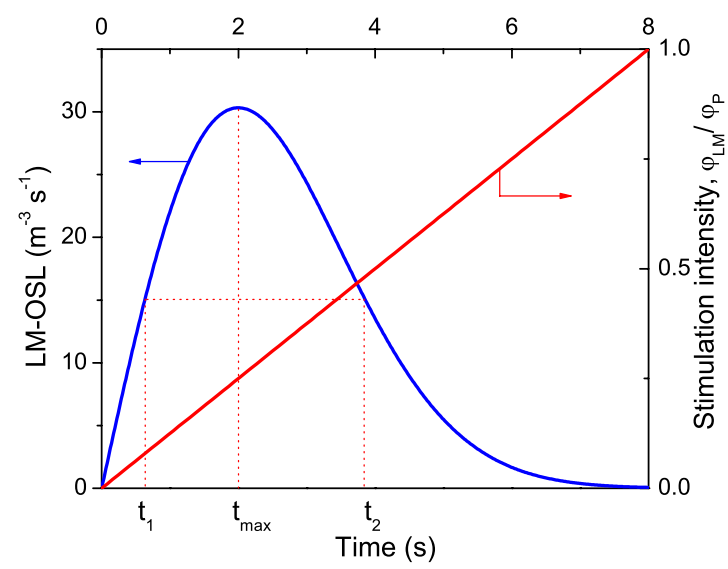

FIG. 1. (Color online) Optically stimulated luminescence signal (14) under linearly increasing intensity (12) with $\beta=2 \mathrm{~s}^{-1}, P$ $=8 \mathrm{~s}$, and $n_{0}=100 \mathrm{~m}^{-3}$.

$$
I_{\text {pseudo-LM }}(t)=\frac{1}{P} t I_{\mathrm{CW}}\left(t^{\prime}\right),
$$

with

$$
t^{\prime}=\frac{1}{2 P} t^{2}
$$

In the determination of $I_{\mathrm{CW}}\left(\frac{1}{2 P} t^{2}\right)$, one should realize that in most experiments the OSL signal is not measured continuously but is digitized, i.e., recorded over finite time intervals (channels). For a specific time $t=t_{1}$, in the middle of a channel there is-in general-no corresponding channel of which the middle precisely corresponds to $\frac{1}{2 P} t_{1}^{2}$. To find $I_{\mathrm{CW}}\left(\frac{1}{2 P} t_{1}^{2}\right)$, one has to logarithmically interpolate (or linear on $\ln I_{\mathrm{CW}}$ ) between the $I_{\mathrm{CW}}$ signals of adjacent channels (the channels closest to $\frac{1}{2 P} t_{1}^{2}$ ).

Our transformation [Eq. (15)] differs from Bulur's,${ }^{14}$ who proposed that Eq. (14) can be obtained by a conversion of the $I_{\mathrm{CW}}$ signal through a transformation of the time $t$ into $u$ $=\sqrt{2 t P}$ with $P(\mathrm{~s})$ as the stimulation period. This leads to a similar equation as Eq. (15) but now in the $u$ domain,

$$
I_{\text {pseudo-LM }}(u)=\frac{1}{P} u I_{\mathrm{CW}}\left(\frac{1}{2 P} u^{2}\right) .
$$

The transformation proposed here [Eq. (15)] is found by interpolation, whereas Bulur's [Eq. (17)] transformation depends on extrapolation. Figure 2 demonstrates that through our interpolation approach, there are more points in the rising part of the fast component. Another advantage of Eq. (15) is that the error propagation inherent to the transformation is reduced.

\section{HM-OSL}

We consider a hyperbolically increasing stimulation intensity according to 



FIG. 2. (Color online) (a) Measured CW-OSL signal from a sedimentary quartz sample NCL-2105096 [see Wallinga and Bos (Ref. 26) for details]. The inset shows the signal on a lin-lin scale. (b) Two pseudo-LM-OSL signals derived by the transformation of the CW-OSL signal. The transformation proposed in this study [Eq. (15)] is shown in red and the Bulur (Ref. 14) transformation [Eq. (17)] in black. The readout period was too short to completely deplete the trap corresponding to the slow component so that only the rising part of this component can be seen in the pseudo-OSL signal. Note in the lower figure the difference in number of points on the left-hand side of the peak maximum and the difference in noise in the late part of the spectrum.

$$
\varphi_{\mathrm{HM}}(t)=\frac{\delta t}{1+\delta t} c \varphi_{P}
$$

with $\delta\left(\mathrm{s}^{-1}\right)$ as an HM stimulation-rate parameter and $c$ as a constant defined by $c=(1+\delta P) / \delta P$. The dimensionless constant $c$ has been chosen such that for any value of $\delta$ at the end of the stimulation period $P(\mathrm{~s})$, the intensity is equal to $\varphi_{P}$ or $\varphi_{\mathrm{HM}}(P)=\varphi_{P}$. Note that for small values of $\delta(\delta t \ll 1)$, the stimulation function $\varphi_{\mathrm{HM}}(t)$ is almost linear in $t$ (approaching LM-OSL), while for large values of $\delta(\delta t \gg 1)$ the stimulation becomes a step function (approaching CW-OSL) (see Fig. 3). We define the HM decay-rate parameter $\gamma\left(\mathrm{s}^{-1}\right)$ as

$$
\gamma=\sigma c \varphi_{P}
$$

The HM-OSL signal follows from Eq. (9) with $f(t)=\delta t /(1$ $+\delta t), \varphi_{c}=c \varphi_{P}$, and using Eq. (19):



FIG. 3. (Color online) Optically stimulated luminescence signal (20) under hyperbolically increasing intensity (18) with $\delta=\gamma$ $=2 \mathrm{~s}^{-1}, P=5 \mathrm{~s}$, and $n_{0}=100 \mathrm{~m}^{-3}$. The increase in the stimulation intensity with stimulation-rate parameter $\delta=0.02 \mathrm{~s}^{-1}, \delta=0.4 \mathrm{~s}^{-1}$, and $\delta=20 \mathrm{~s}^{-1}$ (dashed curves) are shown as well.

$$
I_{\mathrm{HM}}(t)=n_{0} \gamma \delta t \exp \left\{\left(\frac{\gamma}{\delta}-1\right) \ln (1+\delta t)-\gamma t\right\} .
$$

The function has an asymmetric peak shape with a fast-rising left-hand side and a relatively slow decreasing right-hand side (see Fig. 3). The intensity reaches its maximum at

$$
t_{\max }=\frac{1}{\sqrt{\gamma \delta}}
$$

with a value

$$
I_{\max }=n_{0} \sqrt{\gamma \delta} \exp \left\{\left(\frac{\gamma}{\delta}-1\right) \ln \left(1+\sqrt{\frac{\delta}{\gamma}}\right)-\sqrt{\frac{\gamma}{\delta}}\right\} .
$$

Pseudo-HM-OSL. The pseudo-LM-OSL signal follows from Eq. (10):

$$
I_{\text {pseudo-HM }}(t)=\frac{\delta t}{1+\delta t} I_{\mathrm{CW}}\left(t^{\prime}\right)
$$

with

$$
t^{\prime}=t-\frac{1}{\delta} \ln (1+\delta t)
$$

Here we assume that $c \varphi_{P}$ is equal to the intensity as in case of CW-OSL, i.e., $c \varphi_{P}=\varphi_{\mathrm{CW}}$. This rather complicated transformation simplifies if we choose the stimulation-rate parameter $\delta$ equal to the decay-rate parameter $\gamma$. In that case, Eq. (20) reduces to

$$
I_{\mathrm{HM}}(t)=n_{0} \gamma^{2} t \exp (-\gamma t)
$$

If we assume again $c \varphi_{P}=\varphi_{\mathrm{CW}}$ which implies $\gamma=\alpha$, Eq. (25) can be derived from the signal under continuous stimulation (4) via 


$$
I_{\text {pseudo-HM }}(t)=-t \frac{d I_{\mathrm{CW}}(t)}{d t} .
$$

The intensity starts from zero at $t=0$ and exhibits a maximum at $t_{\max }=1 / \alpha$. The intensity at the maximum is $I_{\max }$ $=n_{0} \alpha / e$, the FWHM $=2.446 / \alpha$ and the asymmetry $\tau=0.686$. It should be realized that Eq. (26) is only valid in case $\delta$ $=\gamma$ and cannot be measured for a multicomponent signal since in a real experiment the stimulation-rate parameter can be chosen to be equal to the decay rate of only one of the components.

\section{EM-OSL}

We consider an exponentially increasing intensity according to

$$
\varphi_{\mathrm{EM}}(t)=\varphi_{0} e^{\nu t},
$$

with $\varphi_{0}\left(\mathrm{~m}^{-2} \mathrm{~s}^{-1}\right)$ as the stimulation intensity at $t=0$ and $\nu\left(\mathrm{s}^{-1}\right)$ as the EM stimulation-rate parameter. We define $\mu\left(\mathrm{s}^{-1}\right)$ as the EM decay-rate parameter according to

$$
\mu=\sigma \varphi_{0} .
$$

The EM-OSL signal follows from Eq. (9) with $f(t)$ $=\exp (\nu t), \varphi_{c}=\varphi_{0}$, and using Eq. (28):

$$
I_{\mathrm{EM}}(t)=n_{0} \mu \exp \left[\nu t+\frac{\mu}{\nu}\{1-\exp (\nu t)\}\right] .
$$

After some rearrangement, this equation can be written as

$$
I_{\mathrm{EM}}(t)=I_{\max } \exp \left[1+\nu\left(t-t_{\max }\right)-e^{\nu\left(t-t_{\max }\right)}\right],
$$

with

$$
\begin{aligned}
& t_{\max }=\frac{1}{\nu} \ln \frac{\nu}{\mu}, \\
& I_{\max }=g \frac{n_{0} \nu}{e},
\end{aligned}
$$

where

$$
g=\exp \left\{\exp \left(-\nu t_{\max }\right)\right\} .
$$

The function has an asymmetric peak shape with a long slowly rising left-hand side and a relatively rapidly falling right-hand side (see Fig. 4). The asymmetry $\tau=0.403$ and the $\mathrm{FWHM}=2.446 / \nu$. Note that the FWHM is independent of $\sigma$, which implies that all OSL components have the same FWHM. In contrast to LM- and HM-OSL, the intensity at $t=0$ is not equal to zero. As long as $\nu \gg \mu$ (as is the case in the example of Fig. 4), the OSL signal at $t=0$ is very small compared to $I_{\max }$ and the factor $g$ is very close to unity. If $\nu$ has a value of the same order as $\mu$, the intensity at $t=0$ is significant compared to $I_{\max }$ (see solid curve in Fig. 5). In this case, the factor $g$ strongly deviates from unity. It suggests the presence of a virtual OSL signal for the time $t<0$. If we set $g=1$ (see Fig. 5 dashed curve) the time-integrated OSL signal, e.g., integrated from $t=0$ to $t=+\infty$ will not be equal to $n_{0}$ [the constraint required by Eq. (6)]. However, it can be shown that the area under the whole dashed curve,

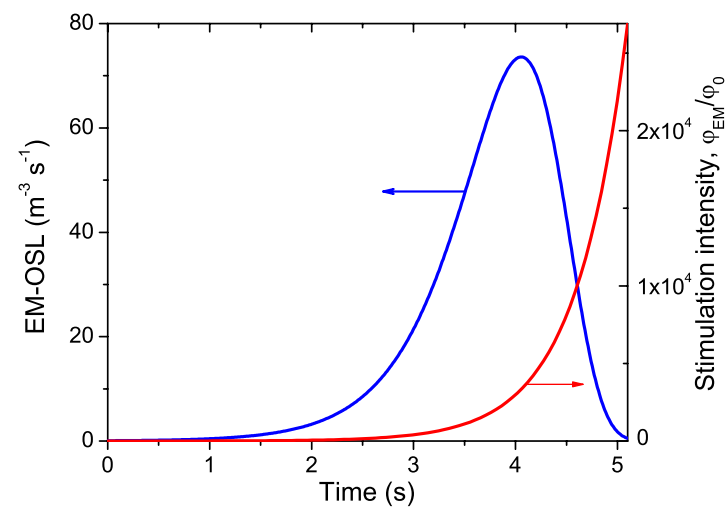

FIG. 4. (Color online) Optically stimulated luminescence signal (29) under exponentially increasing intensity (27) with $\nu=2 \mathrm{~s}^{-1}$, $\mu=6 \times 10^{-4} \mathrm{~s}^{-1}$, and $n_{0}=100 \mathrm{~m}^{-3}$.

e.g., integrated from $t=-\infty$ to $t=+\infty$ is equal to $n_{0}$. The factor $g$ can therefore be interpreted as the factor that corrects or compensates for the time-integrated virtual OSL signal. If $g=1$ (which can be approximated very closely since $\nu$ is an experimental parameter) then the maximum of the HM-OSL signal is also independent of the photoionization cross section. This implies that for a multicomponent OSL signal at a given EM intensity, all components only differ in the position of the maximum determined by $\mu_{i}$ and in a vertical scaling factor determined by $n_{0, i}$.

Pseudo-EM-OSL. The pseudo-EM-OSL signal follows from Eq. (10):

$$
I_{\text {pseudo-EM }}(t)=e^{\nu \mathrm{t}} I_{\mathrm{CW}}\left(t^{\prime}\right)
$$

with

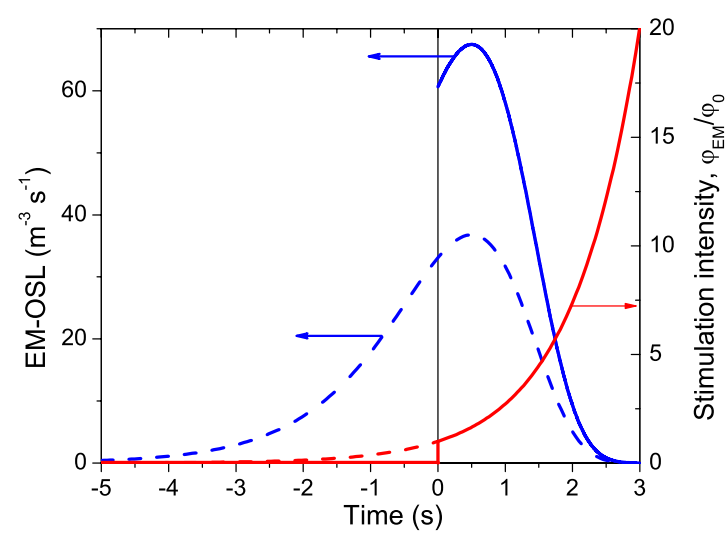

FIG. 5. (Color online) The blue solid curve shows the optically stimulated luminescence signal under exponentially increasing intensity (red solid curve) according to Eq. (30) with $\nu=1 \mathrm{~s}^{-1}, \mu$ $=0.607 \mathrm{~s}^{-1}$, and $n_{0}=100 \mathrm{~m}^{-3}$. In this case, $t_{\max }=0.5 \mathrm{~s}$ and $g=1.8$. The area under the blue solid curve integrated from $t=0$ to $t=\infty$ is

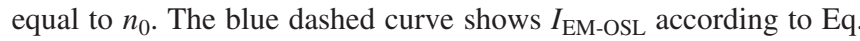
(30) with the same values for $\nu, \mu$, and $n_{0}$ but now with $I_{\max }$ $=n_{0} \nu / e$, i.e., with the factor $g=1$. The shapes of the solid and dashed blue curves are identical for $t \geq 0$. They only differ by a scaling factor $g$. The dashed blue curve represents a hypothetical OSL signal for exponential stimulation intensity according to the dashed red curve for $t<0$ and the solid red curve for $t \geq 0$. It can be shown that the area under the dashed blue curve integrated from $t$ $=-\infty$ to $t=+\infty$ is also equal to $n_{0}$. 


$$
t^{\prime}=\frac{1}{\nu}\left(e^{\nu t}-1\right)
$$

An interesting case appears if we apply a slightly different time transformation $t \rightarrow t^{\prime \prime}=(1 / \nu) e^{\nu t}$. Then the transformed signal becomes

$$
\begin{aligned}
I_{\text {pseudo-EM }}(t)= & e^{\nu t} I_{\mathrm{CW}}\left(t^{\prime \prime}\right) \\
= & e^{\nu t} n_{0} \alpha \exp \left(-\frac{\alpha}{\nu} e^{\nu t}\right) \\
= & \frac{n_{0} \nu}{e} \exp \left\{1+\nu\left(t-\frac{1}{\nu} \ln \frac{\nu}{\alpha}\right)\right. \\
& \left.-\exp \left[\nu\left(t-\frac{1}{\nu} \ln \frac{\nu}{\alpha}\right)\right]\right\} .
\end{aligned}
$$

This signal is equivalent to Eq. (30) with $t_{\max }$ $=(1 / \nu) \ln (\nu / \alpha)$ and $I_{\max }=n_{0} \nu / e$ and $g=1$. With the transformation $t \rightarrow t^{\prime \prime}$, we again produce a pseudo-EM-OSL signal; but to obtain the total OSL signal we have to integrate from $t=-\infty$ to $t=+\infty$. If we take the exponential stimulation rate to be $\nu=1 \mathrm{~s}^{-1}$, Eq. (36) can be written as

$$
I_{\text {pseudo-EM }}(\ln t)=t I_{\mathrm{CW}}(t) .
$$

From Eq. (37) it follows that if we plot the product $t \cdot I_{\mathrm{CW}}$ as a function of the natural logarithm of the time, the same curve is obtained as under exponentially increasing intensity with stimulation-rate parameter $\nu=1 \mathrm{~s}^{-1}$.

\section{RM-OSL}

In the foregoing, we have seen that in a number of cases an increasing stimulation intensity produces a peak-shaped OSL curve. However, this is not necessarily the case for any monotonically increasing stimulation intensity. We illustrate this with a reciprocal stimulation function according to

$$
\varphi_{R M}(t)=\frac{1}{1-\varepsilon t} \varphi_{0},
$$

with $\varphi_{0}\left(\mathrm{~m}^{-2} \mathrm{~s}^{-1}\right)$ as the stimulation intensity at $t=0$ and $\varepsilon\left(\mathrm{s}^{-1}\right)$ as the RM stimulation-rate parameter [see Fig. 6(a)]. We define $\eta\left(\mathrm{s}^{-1}\right)$ as the RM decay-rate parameter according to

$$
\eta=\sigma \varphi_{0} .
$$

The charge-carrier concentration as a function of time follows from Eq. (8) with $f(t)=1 /(1-\varepsilon t), \varphi_{c}=\varphi_{0}$, and using Eq. (39):

$$
n(t)=n_{0}(1-\varepsilon t)^{\eta / \varepsilon} .
$$

The RM-OSL signal follows from Eq. (9):

$$
I_{\mathrm{RM}}(t)=n_{0} \eta(1-\varepsilon t)^{\eta / \varepsilon-1} .
$$

The behavior of the RM-OSL signal strongly depends on the ratio $\eta / \varepsilon$. If $\eta / \varepsilon<1$, the OSL signal is monotonically increasing, while for $\eta / \varepsilon>1$ the signal is monotonously decreasing [see Fig. 6(b)]. A special case occurs if $\eta / \varepsilon=1$. Then the increase in the OSL signal due to the increase in the


FIG. 6. (Color online) Reciprocally increasing intensity (38) for various stimulation rates $\varepsilon$ in $\mathrm{s}^{-1}$ as indicated (upper figure) and the corresponding simulated RM-OSL signal (lower figure) according to Eq. (41) with $n_{0}=100 \mathrm{~m}^{-3}$ and $\eta=1 \mathrm{~s}^{-1}$.

stimulation intensity is exactly compensated by the decrease due to the trap emptying; in other words, the signal is constant. If there is only one trap involved, the RM mode can be used to test the assumptions on which Eq. (1) is based. By varying the stimulation-rate parameter $\varepsilon$ from $\langle\eta$ to $>\eta$, the OSL signal must change from decreasing to an increasing signal. For $\varepsilon=\eta$, the OSL signal should be constant.

In a real experiment with an RM stimulation mode, one should realize that it is not possible to fully empty the trap. To fully empty the trap, i.e., $n(t)=0$, according to Eq. (40) the increase in intensity must last up to $t=1 / \varepsilon$. This means according to Eq. (38), an increase in the intensity to infinity. In practice the RM-OSL signal will only be described by Eq. (41) as long as the light source is able to produce an increase in intensity dictated by Eq. (38).

Pseudo-RM-OSL. The pseudo-RM-OSL signal follows from Eq. (10):

$$
I_{\text {pseudo-RM }}(t)=\frac{1}{1-\varepsilon t} I_{\mathrm{CW}}\left(t^{\prime}\right),
$$

with 


$$
t^{\prime}=\frac{1}{\varepsilon} \ln \frac{1}{1-\varepsilon t} .
$$

If the stimulation-rate parameter $\varepsilon$ is equal to the decay-rate parameter, the OSL signal will be constant. Another particular case occurs if $\alpha / \varepsilon=2$. Then the RM-OSL signal is a straight line with a negative slope equal to $\frac{1}{2} n_{0} \alpha^{2}$.

\section{DISCUSSION}

In Table I the most important equations are summarized. We have shown that depending on the stimulation mode, the OSL signal can be monotonously increasing, monotonously decreasing, shows a peak shape, or be constant. For all nonconstant stimulation modes investigated, we defined a stimulation-rate parameter $(1 / P, \delta, \nu$, and $\varepsilon$; column 2 in Table I) which describes the rate $\left(\mathrm{s}^{-1}\right)$ at which the stimulation intensity increases. The value of this parameter can be selected for an experiment. The resulting OSL signal depends on the stimulation-rate parameter and on a decay-rate or detrapping parameter (column 3 in Table I). Note that in case of a peak-shaped OSL signal, the position of the maximum is determined by either the product or the ratio of the stimulation-rate and the decay-rate parameter (column 5 in Table I). The decay-rate parameter is proportional to the photoionization cross section, which is a property of the particular defect within the material and depends on the stimulation wavelength. In all cases, the trapped-charge concentration at $t=0\left(n_{0}\right)$ assumed to be related to the absorbed dose is a scaling factor (column 4 in Table I).

\section{A. Optically stimulated luminescence versus thermoluminescence}

OSL and TL differ in the type of energy by which the trapped-charge carriers are freed from their trapping centers. In case of TL, heat is transferred to the sample; in case of OSL the sample is exposed to photons of a specific energy. In the simple one-trap one-recombination-center case, with no retrapping (first-order kinetics) the luminescence intensity can be described by a generalized form of Eq. (1),

$$
I(t)=-\frac{d n}{d t}=p n
$$

with $p$ as the probability per unit time that charge carriers escape from their trap. In case of thermal stimulation, this probability is given by Arrhenius' relation

$$
p_{\mathrm{TL}}(t)=s \exp \left(-\frac{E}{k T(t)}\right),
$$

with $s\left(\mathrm{~s}^{-1}\right)$ as the frequency factor, $E(\mathrm{eV})$ as the trap depth, $k(\mathrm{eV} / \mathrm{K})$ as Boltzmann's constant, and $T(\mathrm{~K})$ as the absolute temperature. Under hyperbolic heating, ${ }^{21}$ the temperature increases according to

$$
\frac{1}{T}=\frac{1}{T_{0}}-b t,
$$

with $T_{0}$ as the temperature at $t=0$ and $b\left(\mathrm{~K}^{-1} \mathrm{~s}^{-1}\right)$ as a heating-rate constant. Strictly speaking Eq. (46) is a recipro-

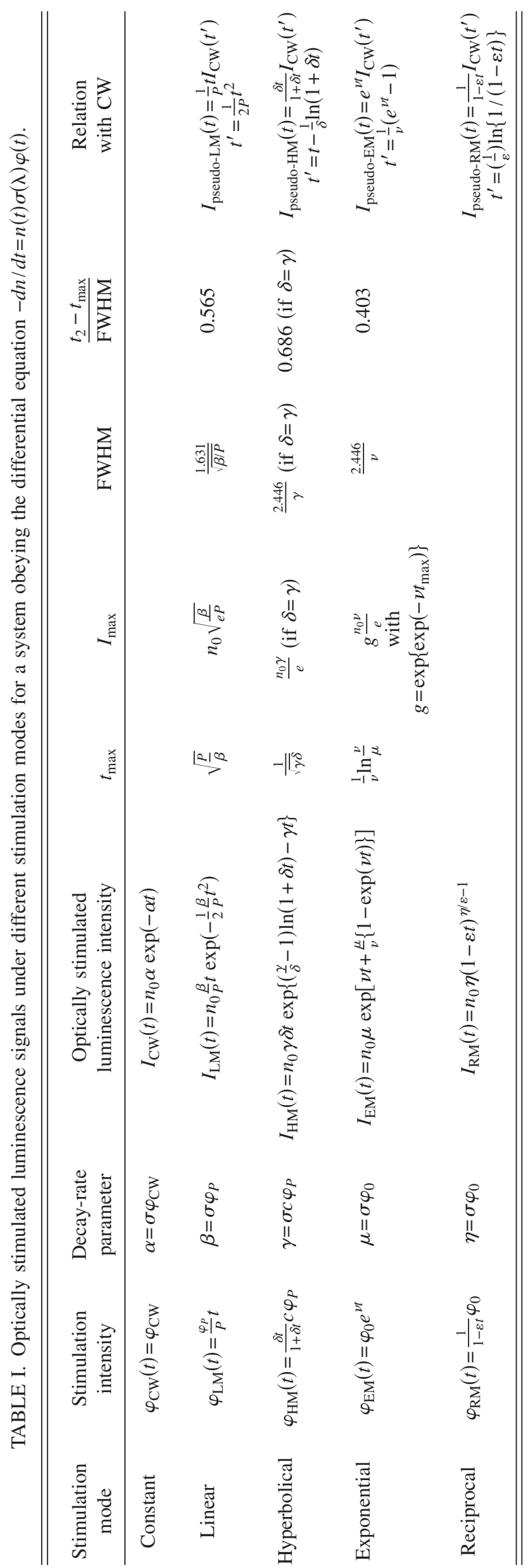


cal function, ${ }^{22}$ but in the literature this heating mode is referred to as hyperbolic heating. ${ }^{20,23-25}$ If we substitute Eq. (46) into Eq. (45), we arrive with a function that can be written as

$$
p_{\mathrm{TL}}(t)=\mu^{\prime} e^{\nu^{\prime} t},
$$

with $\mu^{\prime}=s \exp \left(-E / k T_{0}\right)$ and $\nu^{\prime}=E b / k$.

In the case of optical stimulation, the probability per unit time that trapped-charge carriers escape from their trap under exponentially modulated stimulation is given by

$$
p_{\mathrm{OSL}}(t)=\sigma \varphi_{\mathrm{EM}}(t)=\mu e^{\nu t} .
$$

Mathematically speaking, Eqs. (47) and (48) are identical and so are the solutions for OSL and TL of Eq. (44). This implies that Eq. (29) can also be used to describe the TL under hyperbolic heating but with the replacement of the parameters $\mu$ and $\nu$ with $\mu^{\prime}$ and $\nu^{\prime}$, respectively. This allows us to interpret the virtual OSL signal (the apparent OSL signal for $t<0$ ) as similar to the TL signal for $T<T_{0}$.

From Eqs. (47) and (48), we infer that the luminescence signal as a function of time under exponentially increasing optical stimulation (EM-OSL) shows the same evolution in time as the luminescence signal under hyperbolically increasing thermal stimulation (HM-TL). This similarity allows another interpretation of the $t \cdot I$ vs $\ln t$ plot often used in phosphorescence and OSL. In their original paper, Randall and Wilkins ${ }^{9}$ interpreted the peak-shaped curve to resemble a TL glow curve. The expression for the pseudo-EM-OSL signal $[$ Eq. (37)] shows that the transformed curve can also be interpreted as resembling an OSL signal obtained under an exponentially increasing stimulation intensity.

\section{B. Effect of stimulation mode on overlap of the components}

Usually an OSL material contains several traps with different photoionization cross sections resulting in an OSL signal with several overlapping components. In this section, we investigate the effect of stimulation mode on the overlap of OSL contributions from individual components. The OSL intensity ratio at time $t$ of two components 1 and 2 with photoionization cross sections $\sigma_{1}$ and $\sigma_{2}$ and initial trappedcharge concentrations $n_{0,1}$ and $n_{0,2}$ follows from Eq. (1):

$$
\frac{I_{\mathrm{OSL}, 1}(t)}{I_{\mathrm{OSL}, 2}(t)}=\frac{n_{1}(t)}{n_{2}(t)} \frac{\sigma_{1}}{\sigma_{2}} .
$$

With the aid of Eq. (9) and using Eq. (7), we find for this ratio

$$
\frac{I_{\mathrm{OSL}, 1}(t)}{I_{\mathrm{OSL}, 2}(t)}=\frac{n_{0,1}}{n_{0,2}} \frac{\sigma_{1}}{\sigma_{2}} \exp \left\{\left(\sigma_{2}-\sigma_{1}\right) \int_{0}^{t} \varphi(\theta) d \theta\right\} .
$$

From Eq. (50) we see that the ratio of intensities of two OSL components depends on (i) the ratio of initial trapped-chargecarrier concentrations, (ii) the ratio of the photoionization cross sections (at wavelength $\lambda$ ), and (iii) the time-integrated stimulation intensity, which is proportional to the cumulative stimulation energy.

It is important to note that the ratio is independent of the way the photons are distributed in time during the stimula- tion period. The stimulation mode $\varphi(t)$ may be different; but as long as the time-integrated number of photons is the same (i.e., the total amount of energy the sample is exposed to) then the ratio of the components remains unaltered. Experimental evidence for this finding for OSL of sedimentary quartz is given by Wallinga et al. ${ }^{26}$ Claims for an improved discrimination of the components using LM-OSL or nonlinear stimulation are therefore not justified in cases where the stimulation is performed at a fixed wavelength. We note that for non-first-order kinetics, a similar derivation can be given resulting in an expression where again $\varphi(t)$ only occurs under the integral sign. So for non-first-order kinetics, the ratio remains unaltered as well.

\section{Effect of transformations on overlap of the components}

A pure pseudo-OSL signal mimics precisely the OSL signal measured under a specific stimulation mode. Since the overlap of the components in a measured OSL signal will not be altered by applying another stimulation mode, as shown in Sec. III B, the same applies to pseudo-OSL signals.

In this section, we investigate whether improved component separation can be obtained through the application of Eq. (26) to a multicomponent OSL signal. In Sec. II B 2, we discussed that this equation only provides a pseudo-HM signal when a single trap contributes to the OSL signal. Here we consider the OSL intensity ratio of two components 1 and 2 with decay rates $\alpha_{1}$ and $\alpha_{2}$ and initial electron concentrations $n_{0,1}$ and $n_{0,2}$, respectively. Under $\mathrm{CW}$ stimulation, this ratio becomes

$$
\left(\frac{I_{1}(t)}{I_{2}(t)}\right)_{\mathrm{CW}}=\frac{n_{0,1} \alpha_{1} \exp \left(-\alpha_{1} t\right)}{n_{0,2} \alpha_{2} \exp \left(-\alpha_{2} t\right)} .
$$

For the transformed CW-OSL signal according to Eq. (26), this ratio becomes

$$
\left(\frac{I_{1}(t)}{I_{2}(t)}\right)_{\text {diff }}=\frac{n_{0,1} \alpha_{1}^{2} \exp \left(-\alpha_{1} t\right)}{n_{0,2} \alpha_{2}^{2} \exp \left(-\alpha_{2} t\right)} .
$$

The difference between the initial and transformed intensity ratios is a factor $\alpha_{1} / \alpha_{2}$; thus the transformation according Eq. (26) to a multicomponent OSL signal was successful in enlarging the component separation. We note that the strength of this approach depends on the difference in photoionization cross section of the traps involved. The larger the difference between $\alpha_{1}$ and $\alpha_{2}$, the greater the increase in component separation through transformation.

The improved peak separation is a consequence of the property that the derivative of an exponential is again an exponential. Further separation can be obtained by repeated differentiation. This brings us to define

$$
I_{\mathrm{diff}, k}(t) \equiv \frac{(-1)^{k}}{k !} t^{k} \frac{d^{k} I_{\mathrm{CW}}(t)}{d t^{k}},
$$

with $k$ as a positive integer. The factor $1 / k$ ! in this equation has been included in the realization that according to Eq. (6) for each component $i$, the time-integrated signal should be equal to the initial concentration of trapped-charge carriers, 


$$
\int_{0}^{\infty} I_{\mathrm{diff}, k, i}(t) d t=n_{0, i} .
$$

Equation (54) can be derived using the equality ${ }^{27}$

$$
\int_{0}^{\infty} t^{k} e^{-\alpha t} d t=\frac{k !}{\alpha^{k+1}} .
$$

For a single component the intensity $I_{\text {diff, } k}(t)$ shows a peak shape with a maximum at $t_{\max }=k / \alpha$. The intensity at the maximum is $I_{\max }=\left\{k^{k} / k !\right\} \times\left\{n_{0} \alpha / e^{k}\right\}$ and the FWHM is proportional in a first-order approximation to $\sqrt{k} / \alpha$. From these expressions, we see that the relative peak width $\left(\mathrm{FWHM} / t_{\max }\right) \sim 1 / \sqrt{k}$, and thus decreases with increasing $k$. This means that for each further differentiation, the overlap of the OSL components decreases and the peaks become more separated. This effect is illustrated in Fig. 7 in which $I_{\mathrm{CW}}$ and $I_{\mathrm{diff}, k}$ are calculated for a three component OSL signal with decay constants differing by a factor of 10 . Besides the improved separation, the application of Eq. (53) to a measured CW-OSL signal also results in the automatic removal of the background since under $\mathrm{CW}$ stimulation the background is constant and the derivative of a constant is zero.

We would like to re-emphasize that for a multicomponent signal, the intensity described by Eq. (53) cannot be measured under hyperbolically modulated stimulation or any other stimulation mode. So it is not a pseudo-OSL signal, but a theoretical function with applications for analysis of a multicomponent CW-OSL signal. Analysis of a multiple exponential function by differentiation has been applied before. Okushi and Tokumaru, ${ }^{28}$ for example, used an equation similar to Eq. (26) for the determination of deep-level parameters in semiconductors. In a separate paper, we have investigated the application of this analysis method to experimental data on sedimentary quartz. ${ }^{29}$

\section{Effect of stimulation mode on the pattern of a multicomponent OSL signal}

The stimulation mode has no effect on the overlap of the different components but does influence the time interval in which a component is readout. The result is that the pattern of a multicomponent OSL signal, i.e., the ratios of $I_{\max }$ of the various components, depends strongly on the stimulation mode. This is illustrated in Fig. 8. Figure 8(a) shows a simulated CW-OSL signal with the parameters values shown in Table II. The decay rates are taken from Jain et al..$^{30}$ and are characteristic for the different components in the OSL signal of natural quartz under blue light $(470 \mathrm{~nm})$ stimulation. With the same parameters, we calculated the OSL signal for stimulation under exponentially increasing intensity [Fig. 8(b)], hyperbolically increasing intensity [Fig. 8(c)], and linearly increasing intensity [Fig 8(d)]. Under EM-OSL, the readout interval is equal for all components and the areas of various components can be compared directly. Components with equal $n_{0}$ show equal $I_{\max }$ and also equal areas in a $I$ vs $t$ plot. So it can immediately be seen that the slow components are much more strongly represented than the fast/medium com-
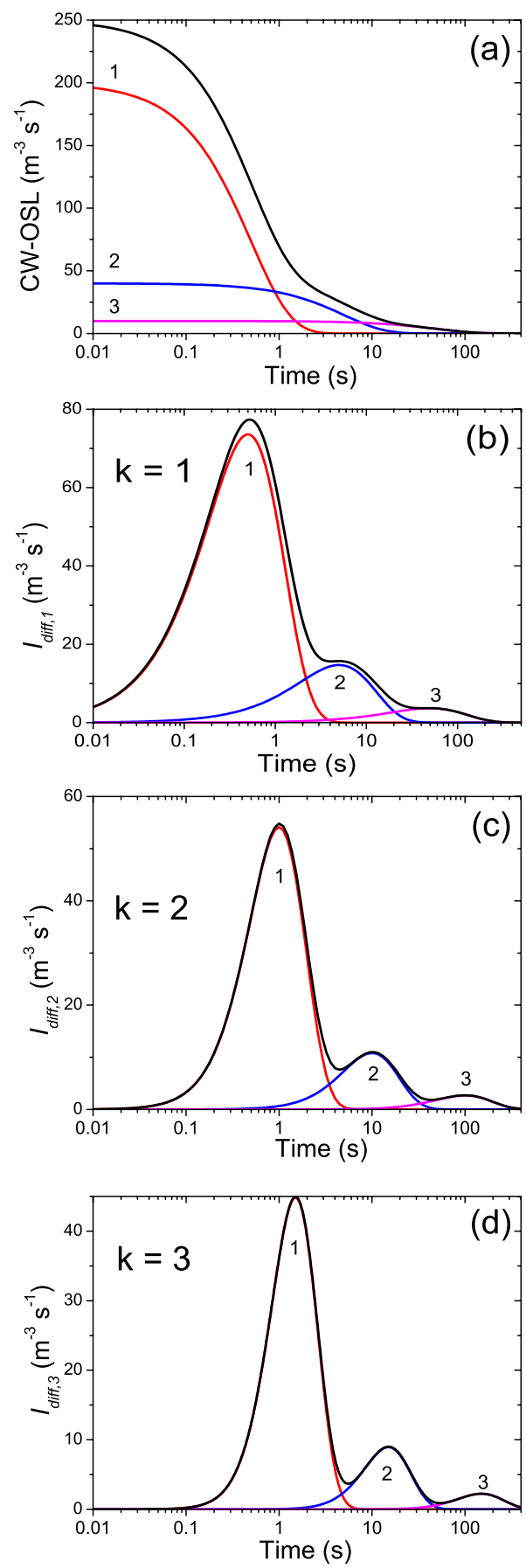

FIG. 7. (Color online) (a) Simulated CW-OSL signal with three components $\left(\alpha_{1}=2 \mathrm{~s}^{-1}, \quad n_{0,1}=100 \mathrm{~m}^{-3} ; \quad \alpha_{2}=0.2 \mathrm{~s}^{-1}, \quad n_{0,2}=200\right.$ $\mathrm{m}^{-3}$; and $\alpha_{3}=0.02 \mathrm{~s}^{-1}, n_{0,3}=500 \mathrm{~m}^{-3}$ ) and the transformed OSL signal according to Eq. (53) for (b) $k=1$, (c) $k=2$, and (d) $k=3$. Note that the peaks become more separated for repeated differentiation (higher $k$ ). As a result, the overlap between the first and second components is reduced and the overlap of the first and third components becomes negligible.

ponents. This cannot be so easily read from the CW-OSL signal on a $I_{\mathrm{CW}}$ vs $\ln t$ plot. The HM-OSL signal [Fig. 8(c)] emphasizes the fast/medium components, while in the LMOSL signal [Fig. 8(d)] both fast/medium and slow components are clearly noticeable. This is a consequence of the fact 



FIG. 8. (Color online) (a) Simulated CW-OSL signal using Eq. (5) with decay rates of the components which are characteristic for natural quartz under blue LED $(470 \mathrm{~nm})$ stimulation during $5000 \mathrm{~s}$ [based on Jain et al. (Ref. 30)]. (b) EM-OSL signal calculated with Eq. (29), with $\nu=2.0 \times 10^{-4} \mathrm{~s}^{-1}$ and $P=2.09 \times 10^{5} \mathrm{~s}$; the insert shows the EM-OSL signal in the time region between $1.2 \times 10^{5} \mathrm{~s}$ and $1.8 \times 10^{5} \mathrm{~s}$ on another scale, (c) HM-OSL signal calculated with Eq. (20), with $\delta=1 \mathrm{~s}^{-1}$ and $P=5008$ s and (d) LM-OSL signal calculated with Eq. (14), with $P=10000 \mathrm{~s}$. The values for the decay rates and $n_{0, i}$ are taken from Table II. The maximum stimulation intensity and the time-integrated number of stimulation photons are equal for all stimulation modes. Where possible the five OSL components $(F=$ fast, $M=$ medium, S1 =slow1, S2=slow2, and S3=slow3) are indicated. Although the pattern of the OSL signals is different, the overlap of the components is equal for all stimulation modes. Advantages of the different representations are discussed in the main text.

that $I_{\max }$ under HM-OSL is proportional to $\beta(=\gamma)$, while under LM-OSL the maximum intensity is proportional to $\sqrt{\beta}$. The LM-OSL signal suggests that the fast/medium components are more strongly represented, but here the areas (in a $I$ vs $\ln t$ plot) cannot be compared directly.

From the CW-OSL signal shown in Fig. 8(a), we derived the transformed OSL signal according to Eq. (53) with $k=1$.

TABLE II. Parameters values for the components in the OSL signal of natural quartz under blue light $(470 \mathrm{~nm})$ stimulation at $125{ }^{\circ} \mathrm{C}$. The decay rates are characteristic; the values for $n_{0}$ vary from sample to sample [from Jain et al. (Ref. 30)].

\begin{tabular}{cccc}
\hline \hline & \multicolumn{2}{c}{$\begin{array}{c}\text { Decay rate } \\
\left(\mathrm{s}^{-1}\right)\end{array}$} & \\
\cline { 2 - 3 } Component & $\alpha=\beta=\gamma$ & $\mu$ & $n_{0}$ \\
(arb. units)
\end{tabular}

The result is shown in Fig. 9. It can be seen that for this transformation the components are more resolved. In particular, the fast and medium components are more separated and the contribution of the slow 3 component under the fast component is negligible.

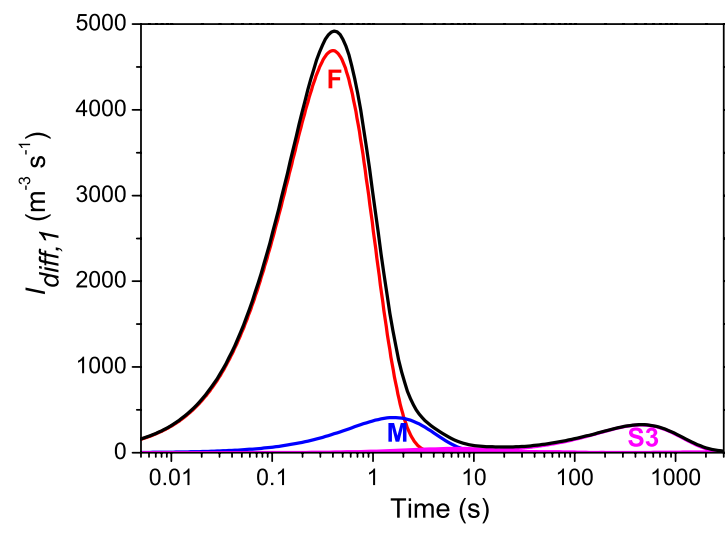

FIG. 9. (Color online) Simulated OSL signal obtained by the transformation of the CW-OSL signal from Fig. 8(a) using Eq. (53) with $k=1$. Note the difference between this figure and Fig. 8(c). 


\section{E. Practical considerations on stimulation mode of preference}

From the viewpoint of separation of the components, there is no preference for a specific stimulation mode. We suggest that CW-OSL should be the method of choice for experimental work: first because CW-OSL is the quickest stimulation mode for readout, second because it gives the highest signal-to-noise ratio (compare the signals in Fig. 8), and third because the background due to the leakage of stimulation light will be constant during the stimulation period. However, when studying an (ultra)fast component, instrumental limitations may make CW-OSL less suitable. Firstly, the sampling rate should be high enough to monitor rapid OSL decays. Reducing the stimulation power may help to solve this issue but may result in long stimulation times to remove slow OSL components. Secondly, the OSL instrument may not be capable of realizing a stimulation intensity change which is a step function, i.e., steep and without undershoot or overshoot (e.g., Ankjaergaard et al. ${ }^{31}$ ). In such cases, HM-OSL provides a good alternative since a hyperbolically increasing intensity starts from zero and is therefore easier to realize while the readout time is only marginally longer than for CW stimulation.

\section{CONCLUSIONS}

Depending on the distribution of stimulation intensity through time (the stimulation mode), the OSL signal can be monotonously increasing, monotonously decreasing, show a peak shape, or be constant. The shape of the OSL signal is determined by the stimulation-rate parameter (the rate at which the stimulation intensity increases) and the decay-rate parameter (determined by the photoionization cross section at a given wavelength of stimulation). We showed that the luminescence signal as function of time under EM-OSL shows the same evolution in time as the luminescence signal under HM-TL. In the case of a multicomponent OSL signal, the overlap of the components is identical for any stimulation mode. This implies that for the separation and identification of the different OSL components there is, in principle, no preference for a specific stimulation mode.

Intensity-modulated OSL signals can be derived from the CW-OSL signal by a time transformation if the stimulation intensity can be described by the product of a timedependent function and a constant intensity. Such a transformed signal (termed as a pseudo-OSL signal) does not contain more physical information than the CW-OSL signal from which it is derived. However, the transformation of a multicomponent CW-OSL curve into a pseudo-OSL curve can be beneficial in visualizing different aspects of the signal since the stimulation mode does influence the time interval in which a component is readout, resulting in different patterns of multicomponent OSL signals. Different transformations highlight different properties and thereby have different applications.

The pseudo-HM-OSL signal for a single exponential can be found by taking the product $t \cdot d I_{\mathrm{CW}} / d t$, but this function provides no pseudo-HM-OSL signal for multiple exponentials. Application of this transformation to a multicomponent CW-OSL signal leads to an improved separation of components with different photoionization cross section. This feature may be of use for investigating trap properties and for isolating the fast OSL component of quartz for dating applications.

\section{ACKNOWLEDGMENT}

The authors would like to thank N. R. J. Poolton for his valuable comments on an earlier version of the manuscript.

\footnotetext{
*a.j.j.bos@tudelft.nl

${ }^{1}$ S. W. S. McKeever, M. Moscovitch, and P. D. Townsend, Thermoluminescence Dosimetry Materials: Properties and Uses (NTP, Ashford, UK, 1995).

${ }^{2}$ L. Bøtter-Jensen, S. W. S. McKeever, and A. G. Wintle, Optically Stimulated Luminescence Dosimetry (Elsevier, Amsterdam, 2003).

${ }^{3}$ D. J. Huntley, D. I. Godfrey-Smith, and M. L. W. Thewalt, Nature (London) 313, 105 (1985).

${ }^{4}$ L. Bøtter-Jensen and A. S. Murray, Radiat. Prot. Dosim. 101, 309 (2002).

${ }^{5}$ J. Trojan-Piegza, J. Niittykoski, J. Hösä, and E. Zych, Chem. Mater. 20, 2252 (2008).

${ }^{6}$ M. S. Akselrod, L. B $\varnothing$ tter-Jensen, and S. W. S. McKeever, Radiat. Meas. 41, S78 (2006).

${ }^{7}$ K. Idri, L. Santoro, E. Charpoit, J. Herault, A. Costa, N. Aillères, R. Delard, J. R. Vailé, J. Fesquet, and L. Dusseau, IEEE Trans. Nucl. Sci. 51, 3638 (2004).

${ }^{8}$ C. Chen, K. L. Teo, T. C. Chong, D. M. Newman, and J. P. Wu, Phys. Rev. B 72, 195108 (2005).

${ }^{9}$ J. T. Randall and M. H. F. Wilkins, Proc. R. Soc. London, Ser. A
}

184, 390 (1945).

${ }^{10}$ R. Chen and V. Pagonis, J. Phys. D: Appl. Phys. 41, 035102 (2008).

${ }^{11}$ R. Chen and N. Kristianpoller, Radiat. Prot. Dosim. 17, 443 (1986).

${ }^{12}$ G. Kitis, I. Liritzis, and A. Vafeiadou, J. Radioanal. Nucl. Chem. 254, 143 (2002).

${ }^{13}$ E. Bulur, Radiat. Meas. 26, 701 (1996).

${ }^{14}$ E. Bulur, Radiat. Meas. 32, 141 (2000).

${ }^{15}$ N. R. J. Poolton, L. Bøtter-Jensen, C. E. Andersen, M. Jain, A. S. Murray, A. E. R. Malins, and F. M. Quinn, Radiat. Meas. 37, 639 (2003).

${ }^{16}$ C. K. Kuhns, N. Agersnap Larsen, and S. W. S. McKeever, Radiat. Meas. 32, 413 (2000).

${ }^{17}$ J. S. Singarayer and R. M. Bailey, Radiat. Meas. 38, 111 (2004).

${ }^{18}$ D. R. Mishra, M. S. Kulkarni, N. S. Rawat, K. P. Muthe, S. K. Gupta, B. C. Bhatt, and D. N. Sharma, Radiat. Meas. 43, 1177 (2008).

${ }^{19}$ A. G. Wintle and A. S. Murray, Radiat. Meas. 41, 369 (2006).

${ }^{20} \mathrm{R}$. Chen and S. W. S. McKeever, Theory of Thermoluminescence and Related Phenomena (World Scientific, Singapore, 1997). 
${ }^{21}$ P. J. Kelley and M. J. Laupnitz, Can. J. Phys. 45, 311 (1967).

${ }^{22}$ P. Müller and J. Teltow, Phys. Status Solidi A 12, 471 (1972).

${ }^{23}$ K. Stammers, J. Phys. E 12, 637 (1979).

${ }^{24}$ G. G. Colvin and W. B. Gilboy, J. Phys. D 18, 283 (1985).

${ }^{25}$ C. Christodoulides, Phys. Status Solidi A 94, 251 (1986).

${ }^{26}$ J. Wallinga, A. J. J. Bos, and G. A. T. Duller, Radiat. Meas. 43, 742 (2008).

${ }^{27}$ I. S. Gradshteyn and I. M. Ryzhik, Table of Integrals, Series, and Products, 4th ed. (Academic, San Diego, 1980), p. 3.33.

${ }^{28}$ H. Okushi and Y. Tokumaru, Jpn. J. Appl. Phys. 19, L335
(1980).

${ }^{29}$ A. J. J. Bos and J. Wallinga, Proceedings of the LED08 Conference, 2008 (to be published) [Radiat. Meas. doi: 10.1016/ j.radmeas.2009.02.005].

${ }^{30}$ M. Jain, A. S. Murray, and L. Bøtter-Jensen, Radiat. Meas. 37, 441 (2003).

${ }^{31}$ C. Ankerjaergaard, M. Jain, R. Kalchgruber, T. Lapp, D. Klein, S. W. S. McKeever, A. S. Murray, and P. Morthekai, Proceedings of the LED08 Conference, 2008 (to be published) [Radiat. Meas. doi: 10.1016/j.radmeas.2009.02.017]. 